\section{OP24 ELICITING CHILDREN'S PERCEPTIONS AND EXPERIENCES OF HEALTH, DIET, PHYSICAL ACTIVITY AND WEIGHT THROUGH THE DRAW, WRITE AND TELL TECHNIQUE}

M Murphy*, W Robertson, R Johnson, FK Boardman. Warwick Medical School, University of Warwick, Coventry, UK

\subsection{6/jech-2017-SSMAbstracts.24}

Background Existing research exploring determinants of childhood obesity tends to overlook the experiential, contextual and cultural basis of such determinants, and lacks methodological features that support children's own framing of issues around obesity. This study aimed to explore children's perceptions and experiences of health, diet, physical activity and weight, with a focus on culture-specific contextual factors that may give rise to childhood obesity, in an ethnically diverse sample.

Methods Children aged 9-10 years were recruited from primary schools in Coventry, UK, with purposive sampling of schools to ensure an ethnically diverse sample. Data were collected through a 'draw, write and tell technique' using semistructured one-to-one interviews at school. Images, text and transcripts were analysed using a thematic framework approach, with themes reviewed and mapped against existing ecological models of health behaviours.

Results To date, six of fifteen invited schools agreed to participate, with 27 children (girls $n=16$ ) successfully recruited from three schools, all of which lived in areas of high deprivation, with $81 \%$ from minority ethnic groups. Recruitment is ongoing to allow for data collection with children from ethnic groups currently under-represented in the sample. Preliminary analysis has identified seven over-arching themes: knowledge, understanding, beliefs, values, motivations, intentions and group-specific experiences. Children had a grasp of the fundamental elements of a healthy diet, physical activity and maintaining a healthy weight but understandings were simplistic and often contradictory. Children viewed health as a personal responsibility, attributed to will-power, but often described external factors affecting their own ability to be healthy e.g. social pressures to indulge and permissive parenting. Positive and negative personal characteristics were assigned to others on the basis of healthfulness and weight. Key motivations for health behaviours were avoidance of the negative social consequences of being overweight, personal success and happiness. Boys and girls differed in their perception of a healthy aesthetic, but culture-specific experiences were largely absent from the data.

Conclusion The findings suggest that childhood obesity interventions could focus on the existing assets that children describe, such as friendships and supportive family environments; emphasising health benefits framed by child-based motivations e.g. personal success and happiness. The lack of culture-specific experiences in children's accounts is inconsistent with other research in ethnically diverse child populations, which may be attributable to the methods used or lack of representation of some ethnic groups. Use of the draw, write and tell technique allowed the research to be child-led, supporting children's own framing of issues.

Health inequalities 2

\section{OP25 EXAMINING THE SITUATED HEALTH AND WELLBEING CHALLENGES OF URBAN FOOD AID PROVISION: A QUALITATIVE PARTICIPATORY STUDY WITH HEALTHCARE PROFESSIONALS IN LONDON}

${ }^{1} \mathrm{C}$ Thompson*, ${ }^{2} \mathrm{D}$ Smith, ${ }^{1} \mathrm{~S}$ Cummins. 'SEHR, London School of Hygiene and Tropical Medicine, London, UK; ${ }^{2}$ Geography and Environment, University of Southampton, Southampton, UK

Note: Presentation moved to gender and family session on friday

\subsection{6/jech-2017-SSMAbstracts.25}

Background Food insecurity is negatively associated with health. Therefore, local food aid provision is crucial to helping protect the health and wellbeing of vulnerable groups. At present, the politics and logistics of this provision are only partially understood and under-theorised. In London, one of the most expensive and equal cities in the world, food insecurity is intensifying. Long-term provision of food aid presents challenges for health maintenance and promotion that are frequently overshadowed by more immediate problems at the local level. This qualitative study examines how London healthcare professionals encounter and seek to address these challenges.

Methods A snowball approach was used to recruit 38 healthcare professionals and food aid coordinators through contacts with participating London food aid organisations. Semi-structured interviews were conducted with all participants. Additionally, observations at a variety of food aid distribution events and stakeholder strategy meetings were used to explore narratives around organisation and health. A critical grounded theory approach to analysis served to identify and situate both the material (physical and mental health) and social (structural inequalities and precarity) issues.

Results Health and wellbeing challenges occur at both the frontline and organisational levels. Participants explained that the mechanisms of addressing (dietary) health via food aid referral are not straightforward. Household interaction with food aid typically occurs during episodes of crisis, which prompt them to turn to healthcare professionals. Food insecurity is rarely the 'presenting problem' and is embedded within a suite of stressors. Frontline healthcare professionals have to deconstruct complex accounts of poverty and find ways to intervene. Offering food aid referral is just one way of doing this. At the organisational level, a culture of precarity makes it extremely difficult to provide consistently nutritious food aid that caters to a reasonable range of medical and religious dietary restrictions. Fluctuating sources of funding, food donation and staffing are obstacles to the implementation of dietary guidelines and the monitoring of nutritional content.

Conclusion Community food aid is undermined by precarity and a lack of structural support, making health and wellbeing concerns difficult to prioritise. Healthcare professionals and food aid coordinators are responding to this in imaginative ways, such as pooling resources, sharing expertise and best practice, and strategizing across sectors and communities. 